Archives

2| 1988

Varia

\title{
Enquête sur la reproduction sociale en milieu rural en Europe, XVIIIe-Xx ${ }^{\mathrm{e}}$ siècles
}

Joseph Goy

\section{OpenEdition \\ Journals}

\section{Édition électronique}

URL : http://journals.openedition.org/ccrh/2944

DOl : $10.4000 /$ ccrh. 2944

ISSN : $1760-7906$

Éditeur

Centre de recherches historiques - EHESS

Édition imprimée

Date de publication : 15 avril 1988

ISSN : 0990-9141

\section{Référence électronique}

Joseph Goy, «Enquête sur la reproduction sociale en milieu rural en Europe, XVIIIe-XXe siècles », Les Cahiers du Centre de Recherches Historiques [En ligne], 2 I 1988, mis en ligne le 13 avril 2009, consulté le 20 avril 2019. URL : http://journals.openedition.org/ccrh/2944 ; DOI : 10.4000/ccrh.2944

Ce document a été généré automatiquement le 20 avril 2019.

Article L.111-1 du Code de la propriété intellectuelle. 


\title{
Enquête sur la reproduction sociale en milieu rural en Europe, XVIIIe-XX ${ }^{e}$ siècles
}

\author{
Joseph Goy
}

1 Dans les travaux conduits depuis une vingtaine d'années, l'étude de la transmission par l'héritage et la succession a toujours été utilisée comme le meilleur révélateur des différentes formes d'organisation sociale dans les sociétés paysannes, chacun tendant à présenter le système qu'il avait mis au jour comme une sorte d'apport théorique décisif à l'anthropologie et donc à l'histoire des sociétés rurales.

Essayons de faire le point sur ces résultats :

3 1. À propos du rapport entre mode de transmission des biens et structures familiales, la coupure entre les deux France - égalitaire et inégalitaire - est bien confirmée. Nous savons également que la structure des groupes domestiques est rarement immuable, qu'elle peut varier avec les époques, le statut social, le cycle de vie et les besoins de l'exploitation. Nous savons aussi que deux systèmes familiaux distincts s'opposent : celui qui privilégie la maison, la lignée domestique et l'exploitation en les rendant indissociables, et celui de la parenté qui autorise la division du patrimoine et de l'exploitation, l'essentiel n'étant pas la transmission du même patrimoine, mais plutôt les relations et les solidarités entre les parents et les alliés.

Beaucoup ont pensé que les alliances matrimoniales étaient au centre du dispositif et qu'elles seraient peut-être pré-déterminées par les modes de transmission des biens, la circulation des dots entre les maisons à l'image de celle des femmes dans les sociétés "élémentaires ». Mais à la clarté, donc à une bonne lisibilité des systèmes inégalitaires s'opposeraient des systèmes égalitaires dans lesquels les cycles d'alliance ne se répéteraient pas et qui connaîtraient une mobilité géographique assez importante. La question de la préservation, de l'intégrité du patrimoine familial séparerait, en gros, les deux systèmes.

2. Il est donc nécessaire de s'interroger sur ce que deviennent les exploitations dans les zones où on ne les transmet pas intégralement à un seul héritier. Cherche-t-on, par 
diverses astuces, à corriger les effets diviseurs de l'égalitarisme ? Dans quelques régions (en Bourgogne du Nord, en Normandie, dans le Jura) il peut arriver que l'on triche avec la tradition et que l'on transmette à un héritier préférentiel. Mais grâce aux travaux de Ségalen, Barthélémy, Augustin, nous savons que l'égalité des partages ne s'oppose pas toujours à la survie des groupes domestiques.

Par la mobilité des ménages et l'utilisation du fermage qui permettent de remodeler sans cesse les exploitations, on est alors conduit à distinguer patrimoine foncier et exploitation agricole, héritage et succession, transmission des biens et transmission des statuts.

7 Il y aurait bien deux stratégies d'apparence contradictoire : celle qui privilégierait le rassemblement du patrimoine dispersé par des alliances entre propriétaires voisins ; celle qui s'intéresserait surtout aux droits d'usage et qui redistribue sans cesse les terres entre héritiers, sous le contrôle de la parentèle.

8 Mais notre perplexité s'accroît encore: car si les sociétés à transmission intégrale forment un ensemble cohérent, homogène, les systèmes égalitaires font coexister des pratiques très différentes :

- Corse : droits égaux sur des patrimoines indivisibles ;

- Bretons du Morbihan : partage effectif ;

- Paysans du Jura : partage égalitaire entre les garçons ;

- Paysans de la région d'Auxerre : intéressement surtout à la transmission de la maison $\mathrm{d}$ 'habitation et location des terres dont ils ont besoin.

Ces trois exemples sont simplement indicatifs.

Bref, nous savons désormais qu'il n'y a pas adéquation entre l'égalité entre les sexes, l'autorité du père, la plus ou moins grande égalité des systèmes d'héritage et le degré plus ou moins élevé de modernisation agricole.

Devant les richesses de la diversité française, nous pensons qu'il ne sert à rien de multiplier les monographies mais qu'il serait infiniment plus utile de confronter nos recherches monographiques non plus dans un cadre national mais dans un cadre européen.

\section{Pourquoi le cadre européen ${ }^{1}$}

Parce que les formes repérées en France, en Espagne ou en Suisse, par exemple, apparaissent extensibles à l'espace européen : dans une thèse de doctorat récente, qui va être très bientôt publiée, Georges Augustin s'est intéressé à la perpétuation des groupes domestiques dans les sociétés paysannes européennes, (c'est le titre).

En bon ethnologue, il s'est davantage intéressé aux conditions d'existence des systèmes successoraux et à leur rôle dans la transmission des exploitations qu'à la vision du monde qui les justifie et les anime. Son hypothèse principale est que les pratiques successorales paysannes font référence, en Europe, à des conceptions différentes de la famille et de la propriété, et que les systèmes à maison et les systèmes à parentèle constitueraient une bonne grille d'analyse pour l'ensemble européen, qui comprendrait trois aires principales :

- une aire des systèmes inégalitaires à maison,

- une aire des systèmes à parentèle égalitaire,

- une aire des systèmes lignagers complexes. 
Mais cette classification sommaire est corrigée par deux constats. Le premier selon lequel la forme qui domine en Europe est mixte : c'est une solution de compromis. Elle emprunte aux trois systèmes et se révèle la plus délicate à interpréter. Le second, c'est qu'une tendance générale, perceptible depuis le début du siècle, tend à généraliser le cas des systèmes à maison.

$\mathrm{J}$ 'en arrive à la deuxième raison du lancement d'une enquête européenne : le moment apparait opportun car une enquête récente de juristes français montre que la plupart des pays d'Europe ont soit pris des décisions juridiques en faveur de l'héritier unique et de la préservation des exploitations (c'est le cas de la Suisse, depuis longtemps, de l'Espagne, récemment), soit rêvent de le faire, comme en France, par exemple. J'ajoute que l'Acte Unique de 1992 ne peut que renforcer encore l'emprise de l'Europe Verte sur les mécanismes de préservation et de transmission des exploitations.

L'enquête aurait pour but de comparer les formes et les mécanismes de la reproduction sociale en milieu paysan, d'abord, entre la deuxième moitié du XVIII ${ }^{e}$ siècle et aujourd'hui, à travers l'exploitation agricole, le ménage, la famille et leurs prolongements.

La grille d'analyse pourrait comporter les éléments suivants :

- description des divers systèmes de transmission observés en rapport avec les types d'exploitation rurale et les modes de faire valoir (facteurs géographiques, démographiques, culturels et notamment juridiques) ;

- l'exploitation agricole comme enjeu de la transmission ;

- la création, la perpétuation ou l'amplification des écarts sociaux par la reproduction inégale ;

- l'impact de l'environnement social sur le fonctionnement et l'évolution des systèmes : développement économique et touristique, développement du marché, Ville, Région, Etat.

- Ainsi pourrait-on envisager :

- de remettre au centre de notre réflexion l'exploitation agricole et le groupe domestique envisagés dans toutes leurs relations avec l'environnement spatial, économique, politique, culturel ;

- de faire avancer la question délicate séparant actuellement les ethnologues et les historiens : l'ensemble des phénomènes de reproduction par alliance, la succession et l'héritage correspondent-ils à des conceptions différentes de la parenté et de la famille?

19 Ne faut-il pas, également, examiner d'un peu plus près, comme l'a fait Bernard Derouet, quel est le rapport à la terre des pratiques familiales et successorales, de quel poids pèsent les faits écologiques, économiques et sociaux : l'organisation du travail, les modes d'accès à la terre, les hiérarchies sociales? Bref, les problèmes fonciers ont-ils une incidence durable sur les processus de transmission?

Au total l'enquête souhaiterait aboutir à un enrichissement de l'histoire sociale du monde rural par la combinaison des apports de la sociologie, de l'ethnologie et de l'histoire. 


\section{NOTES}

1. Une première enquête comparative entre la France et le Québec est en cours : elle aboutira en 1990-91. 